\section{Dimensiones de arcos dentarios en niños de 4 a 8 años de edad con diferente estado nutricional. Talara - Piura}

Moreno K, Meneses A, Morzán E. Dimensiones de arcos dentarios en niños de 4 a 8 años de edad con diferente estado nutricional. Talara - Piura. Rev Estomatol Herediana 2004;14(1-2) : 18 - 21

\section{RESUMEN}

El propósito del presente estudio fue evaluar la influencia del factor nutricional sobre las dimensiones de arco. Se seleccionaron 102 varones y mujeres entre 4 y 8 años de edad, de la posta municipal del AAHH "Jesús María” - Talara. Se subdividió según sexo y estado nutricional. Se midieron en modelos de estudio la longitud, perímetro y ancho de arco; encontrándose diferencias estadísticamente significativas $(\mathrm{p}<0.05)$ mediante la prueba de "t" de Student, en el ancho intermolar superior, perímetro de arco inferior en dentición decidua y, longitud de arco inferior en dentición mixta, siendo menores en las niñas con desnutrición crónica. En estado nutricional normal, los varones presentaron mayor ancho intercanino superior e intermolar inferior que las mujeres, ambos en dentición mixta. En niños con desnutrición crónica, el perímetro de arco superior, ancho intermolar superior e inferior con dentición mixta, y el ancho intermolar superior en dentición decidua, fueron mayores en varones que en mujeres.

Palabras clave: ARCADA DENTARIA / ESTADO NUTRICIONAL / DENTICIÓN MIXTA.

Arch dimensions in $\mathbf{4}$ to 8 years children with different nutritional status

ABSTRACT

The aim of this study was to evaluate how nutrition factors influence on arch length measurements. 102 children, 4-8 years old were selected from a small hospital (Piura- Peru). Dental casts were obtained from these children. The sample was divided into two categories: sex and nutritional condition (normal nutrition and chronic malnutrition). Measurements of maxillary and mandibular arch lengths and intercanine and intermolar arch widths were made on the study casts. The results showed that maxillary intermolar arch width and mandibular perimeter in primary dentition, and mandibular length in mixed dentition were significantly smaller in girls with malnutrition (t- test, $\mathrm{p}<0.05$ ). Boys showed maxillary intercanine width and mandibular intermolar width in mixed dentition larger measurements than girls (normal nutrition). Boys also showed maxillary perimeter and mandibular and maxillary intermolar width in mixed dentition, and maxillary intermolar width in primary dentition larger (malnutrition).

Keywords: DENTAL ARCH / NUTRITIONAL STATUS / MIXED DENTITION.
Karina C. Moreno Rodriguez ${ }^{1}$ Abraham Meneses López ${ }^{2}$ Eduardo Morzán Valderrama²

Cirujano Dentista.

2 Docente del Departamento Académico de Estomatología del Niño y del Adolescente. Facultad de Estomatología. Universidad Peruana Cayetano Heredia.

\section{Introducción}

En el Perú, la desnutrición crónica es uno de los mayores problemas de salud pública. La influencia de la desnutrición crónica sobre las dimensiones de los arcos dentarios como factor etiológico de problemas oclusales no ha sido muy estudiada.

El éxito del tratamiento ortodóntico temprano se basa en el conocimiento del desarrollo de la dentición, particularmente se relaciona con la dinámica de formación del arco dentario (1). Bishara et al. (2) en 1995 concluyeron que las mediciones de los arcos dentarios en dentición permanente se podía predecir con mayor exactitud a partir de la dentición mixta, ya que las dimensiones de arco, en especial de la mandíbula, son más estables cuando los incisivos inferiores han erupcionado. En cuanto a las dimensiones de arco, el ancho intercanino aumenta en ambos maxilares por cierre del espacio primate inferior; el cual, en niños aumenta entre los 5 y 10 años, y en las niñas hasta los 9 años de edad y, a los 8.5 años está completo en $85 \%$ de los niños y el 100\% de las niñas(3). En los estudios realizados por Sillman (4), se concluye que el ancho intermolar aumenta $0.5 \mathrm{~mm}$ por año en el maxilar y 0.2mm en la mandíbula, desde la dentición decidua hasta los 14 años en los varones. Las diferencias entre las medidas del ancho intermolar superior y ancho intermolar inferior son mayores en niños con oclusión aceptable que en aquellos con maloclusión Clase II división 1 (5). Howe et al. (6) encontraron que el ancho intermolar en los casos con apiñamiento fue $6 \mathrm{~mm}$ menor que en los casos sin apiñamiento.
La longitud de arco disminuye antes de la erupción de las primeras molares permanentes por cierre de los espacios entre molares deciduos, y aumenta en la maxila durante la erupción de los incisivos permanentes (7). Mills (8) comparó arcos dentarios con y sin apiñamiento encontrando que no había relación entre la severidad del apiñamiento y la longitud de arco en el maxilar o en la mandíbula. El perímetro de arco en la mandíbula alcanza su máximo tamaño con la erupción de los incisivos permanentes y las primeras molares (9). Moorrees et al. (7) encontraron que entre los 5 y 18 años, el perímetro del arco superior aumenta $1.3 \mathrm{~mm}$ en los niños y $0.5 \mathrm{~mm}$ en las niñas, mientras que en la mandíbula hay una disminución de 3.4mm y 4.5mm en niños y niñas respectivamente. El objetivo de este estudio fue comparar 
las dimensiones de arcos dentarios en dentición decidua y dentición mixta entre niños con estado nutricional normal o con desnutrición crónica.

\section{Material y método}

El diseño del estudio fué transversal, descriptivo y comparativo. La muestra comprendió 102 modelos de estudio de niños entre 4 y 8 años de edad que cumplieron los criterios de inclusión.

Los valores registrados para peso y talla permitieron sub agrupar la muestra, utilizando el parámetro de "Talla para la edad” según los estándares sugeridos por la OPS(10) para clasificar el estado nutricional. Se utilizaron las tablas del Instituto de Nutrición y Tecnología de los alimentos de la Universidad de Chile (11,12).

Se clasificaron a los sujetos en:

1 Estado nutricional normal: si la talla que presentaba para su edad, se ubicaba en la mediana o hasta +-1 desviación estándar (10).

2 Desnutrición crónica: se utilizó como criterio de clasificación por debajo de menos dos (-2) desviaciones estándar de la talla que presentaba para su edad (12).

Se recolectaron los datos personales de cada niño y se realizaron exámenes odontológicos, registrándolos en fichas personales. Las medidas de dimensiones de arco en los modelos fueron realizadas con un compás de dos puntas secas y una regla milimetrada. Se registraron las mediciones de: perímetro de arco, longitud de arco, ancho intermolar y ancho intercanino (Fig. 1).

Fig.1. Análisis de medidas de dimensiones de Arco

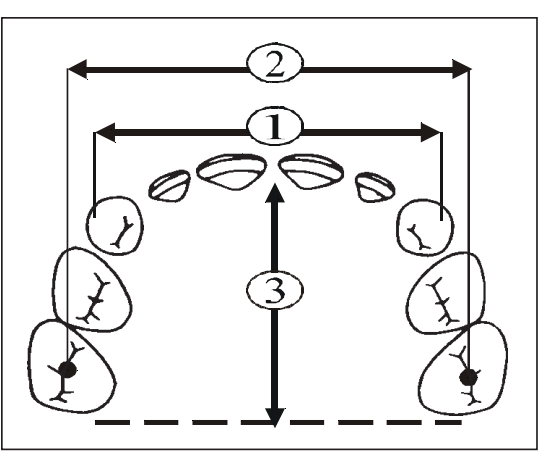

1. Ancho intercanino

2. Ancho intermolar

3. Longitud de arco
Para determinar el error del método, se midió en dos momentos diferentes 10 modelos, (error estándar igual a 0.05). Los resultados fueron procesados estadísticamente mediante el programa SPSS 11.0, las pruebas utilizadas fueron: distribución de frecuencias, medias aritméticas y “t” de Student.

\section{Resultados y discusión}

Se separaron dos subgrupos: dentición decidua y dentición mixta los cuales comparados estadísticamente arro- jaron diferencias no significativas para todas las variables $(\mathrm{p}<0.05)$. La evaluación estadística indicó que existen diferencias estadísticamente significativas $(\mathrm{p}<0.05)$ en las medidas del ancho intermolar superior y perímetro de arco inferior en dentición decidua y, longitud de arco inferior en ambas denticiones, siendo menores en las niñas con desnutrición crónica que en las niñas con estado nutricional normal (Tabla 1 y 2). Esto podría deberse a que una nutrición adecuada es importante para que

Tabla 1. Comparación de valores entre mujeres según Estado Nutricional

\begin{tabular}{|c|c|c|c|c|c|}
\hline & \multicolumn{2}{|c|}{ Estado nutricional normal } & \multicolumn{2}{|c|}{ Desnutrición crónica } & \multirow[t]{2}{*}{$\mathbf{p}$} \\
\hline & $\mathbf{X}$ & d.s & $\mathbf{x}$ & d.s & \\
\hline PAS * & 93.72 & 8.04 & 91.07 & 6.25 & 0.61 \\
\hline pas $* *$ & 72.60 & 3.62 & 70.75 & 3.19 & 0.20 \\
\hline LAS & 24.75 & 1.96 & 23.80 & 1.72 & 0.18 \\
\hline AICS & 31.41 & 2.72 & 30.59 & 1.65 & 0.68 \\
\hline AIMS * & 45.44 & 2.75 & 45.23 & 1.73 & 0.93 \\
\hline aims ** & 40.50 & 1.77 & 38.43 & 1.45 & 0.00 \\
\hline PAI * & 90.56 & 8.64 & 90.92 & 4.48 & 0.16 \\
\hline pai ** & 67.70 & 3.68 & 65.75 & 2.71 & 0.01 \\
\hline LAI & 22.27 & 1.49 & 21.07 & 1.91 & 0.02 \\
\hline AICI & 25.51 & 2.82 & 23.04 & 5.59 & 0.33 \\
\hline AIMI* & 39.72 & 2.40 & 39.34 & 1.79 & 0.77 \\
\hline aimi $* *$ & 34.60 & 1.71 & 33.56 & 1.49 & 0.06 \\
\hline
\end{tabular}

Tabla 2. Comparación de valores entre varones según Estado Nutricional

\begin{tabular}{|c|c|c|c|c|c|}
\hline & \multicolumn{2}{|c|}{ Estado nutricional normal } & \multicolumn{2}{|c|}{ Desnutrición crónica } & \multirow[t]{2}{*}{$\mathbf{p}$} \\
\hline & $\mathbf{X}$ & d.s & $\mathbf{x}$ & d.s & \\
\hline PAS * & 93.82 & 7.45 & 95.92 & 7.67 & 0.31 \\
\hline pas ** & 73.20 & 6.94 & 73.50 & 1.87 & 0.10 \\
\hline LAS & 24.83 & 1.42 & 24.36 & 1.64 & 0.71 \\
\hline AICS & 32.31 & 3.07 & 31.73 & 1.75 & 0.80 \\
\hline AIMS * & 46.88 & 2.54 & 46.76 & 1.48 & 0.59 \\
\hline aims ** & 40.85 & 2.10 & 41.16 & 1.47 & 0.19 \\
\hline PAI* & 90.00 & 10.05 & 92.84 & 9.49 & 0.23 \\
\hline pai ** & 70.80 & 9.43 & 69.50 & 2.16 & 0.37 \\
\hline LAI & 21.79 & 1.44 & 21.92 & 2.26 & 0.83 \\
\hline AICI & 25.51 & 3.00 & 24.89 & 2.60 & 0.88 \\
\hline AIMI* & 41.41 & 2.18 & 41.53 & 1.26 & 0.24 \\
\hline aimi $* *$ & 36.00 & 1.69 & 35.25 & 0.75 & 0.9 \\
\hline
\end{tabular}


Tabla 3. Comparación de valores promedio en sujetos con estado nutricional normal según sexo

\begin{tabular}{|c|c|c|c|c|c|}
\hline & \multicolumn{2}{|c|}{ Mujeres } & \multicolumn{2}{|c|}{ Varones } & \multirow[t]{2}{*}{$\mathbf{p}$} \\
\hline & $\mathbf{X}$ & d.s & $\mathbf{X}$ & d.s & \\
\hline PAS * & 93.72 & 8.04 & 93.82 & 7.45 & 0.89 \\
\hline pas ** & 72.60 & 3.62 & 73.20 & 6.94 & 0.80 \\
\hline LAS & 24.75 & 1.96 & 24.83 & 1.42 & 0.80 \\
\hline AICS & 31.41 & 2.72 & 32.31 & 3.07 & 0.05 \\
\hline AIMS* & 45.44 & 2.75 & 46.88 & 2.54 & 0.11 \\
\hline aims $* *$ & 40.50 & 1.77 & 40.85 & 2.10 & 0.76 \\
\hline PAI * & 90.56 & 8.64 & 90.00 & 10.05 & 0.42 \\
\hline pai ** & 67.70 & 3.68 & 70.80 & 9.43 & 0.36 \\
\hline LAI & 22.27 & 1.49 & 21.79 & 1.44 & 0.07 \\
\hline AICI & 25.51 & 2.82 & 25.51 & 3.00 & 0.34 \\
\hline AIMI* & 39.72 & 2.40 & 41.41 & 2.18 & 0.03 \\
\hline $\operatorname{aimi} * *$ & 34.60 & 1.71 & 36.00 & 1.69 & 0.1 \\
\hline
\end{tabular}

$\mathrm{p}<0.05$

* valor tomado con la primera molar permanente (dentición mixta)

** valor tomado con la segunda molar decidua (dentición decidua)

Tabla 4. Comparación de valores promedio en sujetos con desnutrición crónica según sexo

\begin{tabular}{|c|c|c|c|c|c|}
\hline & \multicolumn{2}{|c|}{ Mujeres } & \multicolumn{2}{|c|}{ Varones } & \multirow[t]{2}{*}{$\mathbf{p}$} \\
\hline & $\mathbf{X}$ & d.s & $\mathbf{X}$ & d.s & \\
\hline PAS * & 91.07 & 6.25 & 95.92 & 7.67 & 0.05 \\
\hline pas ** & 70.75 & 3.19 & 73.50 & 1.87 & 0.06 \\
\hline LAS & 23.80 & 1.72 & 24.36 & 1.64 & 0.19 \\
\hline AICS & 30.59 & 1.65 & 31.73 & 1.75 & 0.20 \\
\hline AIMS* & 45.23 & 1.73 & 46.76 & 1.48 & 0.04 \\
\hline aims $* *$ & 38.43 & 1.45 & 41.16 & 1.47 & 0.02 \\
\hline PAI * & 90.92 & 4.48 & 92.84 & 9.49 & 0.45 \\
\hline pai ** & 65.75 & 2.71 & 69.50 & 2.16 & 0.06 \\
\hline LAI & 21.07 & 1.91 & 21.92 & 2.26 & 0.33 \\
\hline AICI & 23.04 & 5.59 & 24.89 & 2.60 & 0.21 \\
\hline AIMI* & 39.34 & 1.79 & 41.53 & 1.26 & 0.00 \\
\hline $\operatorname{aimi} * *$ & 33.56 & 1.49 & 35.25 & 0.75 & 0.14 \\
\hline
\end{tabular}

\section{$\mathrm{p}<0.05$}

* valor tomado con la primera molar permanente (dentición mixta)

** valor tomado con la segunda molar decidua (dentición decidua)

AICS : ancho intercanino superior, AICI : ancho intercanino inferior

AIMS : ancho intermolar superior, AIMI : ancho intermolar inferior

LAS : longitud de arco superior, LAI

longitud de arco inferior

PAS : perimetro de arco superior, PAI los niños alcancen su potencial genético de crecimiento (11-13). La evaluación según sexo demostró que los varones en dentición mixta presentan mayor ancho intercanino superior y ancho intermolar inferior que las mujeres con estado nutricional normal (Tabla 3). Al comparar los valores promedios de las dimensiones de arco entre varones y mujeres con desnutrición crónica, se observó una diferencia estadísticamente significativa en las medidas del perímetro de arco superior, ancho intermolar superior y ancho intermolar inferior en dentición mixta y el ancho intermolar superior en dentición decidua $(\mathrm{p}<0.05)$, siendo mayor en varones (Tabla 4). En estudios realizados en Lima sobre la influencia del estado nutricional en las dimensiones de los arcos dentarios, se encontró una ligera disminución en todas las dimensiones registradas en los modelos de pacientes con desnutrición crónica $(14,15)$. Nuestros resultados son similares a los de Cohen (16), en cuanto a promedios para el ancho intercanino superior (AICS) y al ancho intercanino inferior (AICI) en dentición mixta y a los de Moorrees et al. (7) en ancho intercanino inferior en dentición mixta (AICI). En el grupo con desnutrición crónica el perímetro de arco superior en dentición mixta (PAS) fue mayor en los varones que en las mujeres. Este hallazgo coincide con lo encontrado en los estudios de Moyers (9), Moorrees et al. (7) y Howe et al. (17) quienes refieren que los niños presentan un mayor perímetro de arco que las niñas. Alfano (18) realizó estudios en cerdos, los cuales demuestran que la desnutrición ocasiona una disminución en el crecimiento y desarrollo mandibular.

\section{Conclusiones}

- La prevalencia de desnutrición crónica encontrada en la muestra fue de $39.21 \%$. No se hallaron diferencias estadísticas según el genero.

- En estado nutricional normal, el ancho intercanino superior (AICS) y el ancho intermolar inferior (AIMI), en dentición mixta fueron significativamente mayores en los varones que en las niñas ( $\mathrm{p} £$ 0.05). Asimis- 
mo, en el grupo con desnutrición crónica, los varones presentaron mayores valores para el perímetro de arco superior (PAS), ancho intermolar superior(AIMS), ancho intermolar inferior (AIMI) en dentición mixta y ancho intermolar superior (aims) en dentición decidua ( $\mathrm{p} £$ 0.05).

- Comparados por estado nutricional, el ancho intermolar superior (aims), el perímetro de arco inferior (pai), en dentición decidua y la longitud de arco inferior en ambas denticiones (LAI), fue mayor significativamente en las mujeres con estado nutricional normal, que en mujeres con desnutrición crónica ( $\mathrm{p} £$ 0.05). No se hallaron diferencias estadísticas entre varones

\section{Referencias bibliograficas}

1. McNamara, William L, Rivas de Montes. Tratamiento ortodóntico y ortopédico en la dentición mixta. Ed. EEUU:Needham Press;1995:55-64.

2. Bishara S, Khadivi P, Jakobsen J. Changes in tooth size - arch length relationships from th deciduos to the permanent dentition: A longitudinal study. Am J Orthod Dentofac Orthop 1995; 108: 607-13.

3. Foley T, Wright G, Weinberger S. Management of lower incisor crowding in the early mixed dentition. J Dent Child 1996;May: 169-74.
4. Sillman JH. Dimensional changes of the dental arches: Longitudinal study from birth to 25 years. Am J Orthod 1964;50:824-842.

5. Bishara S, Jakobsen J, Treder J. Nowak A. Arch width changes from 6 weeks to 45 years of age. Am J Orthod Dentofacial Orthop 1997;11:401-9.

6. Howe RP, Mc Namara JA, Connor KA. AN examination of dental crowding and its relationship to tooth size and arch dimension. Am J Orthodont 1983;83:363-373.

7. Moorrees C, Gron AM, Lebret L, Yen P, Frohlich F. Growth studies of the dentition: a review. Am J Orthodont 1969;55:600-16.

8. Mills L. Arch With, arch length and tooth size in young adult males. Institute of dental research. 1964;34:124-129.

9. Moyers R. Manual de Ortodoncia. Argentina:Editorial Medica Panamericana; 1992:125-130.

10. Organización Panamericana de la Salud. Manual de crecimiento y desarrollo del niño. Washington DC. 1993:25-28.

11. Lopez C. Salud y Nutrición Matrerno Infantil. Colegio Medico del Perú. 1984:76-86.

12. Ministerio de Salud. Situación Nutricional en el Perú. Ministerio de Salud. 1989:305-332.

13. Anderson L. Nutricion y Dieta de
Cooper. 17ma ed; México DF. Edit Interamericana. 1985:315-321.

14. Aranda J. Estudio comparativo dimensional de las segundas molares deciduas, arcos dentarios y características de oclusión entre niños con y sin desnutrición calórica proteica. [Tesis de Bachiller] Lima(Perú): UPCH; 1989.

15. Tomona N. Cambios dimensionales de los arcos dentarios durante la primera fase de dentición mixta en niños peruanos con diferentes estados de nutrición de la ciudad de Lima [Tesis de Especialista] Lima (Perú): UPCH;1999.

16. Cohen J. Growth and development of the dental arches in children. J. Am Dent Assoc. 1940;27:1250 -1260

17. Howe RP, Mc Namara JA, Connor KA. An examination of dental crowding and its relationship to tooth size and arch dimension. Am J Orthodont 1983;83:363-73

18. Alfano M. Diet and nutrition in the etiology and prevention of oral disease. J Dent Res (59) DI:2194202,1980

\section{Dirección de Correspondencia}

Karina C. Moreno Rodriguez

Calle El Palo Santo MzB Lt7. Urb. El Pedregal de Higuereta. Lima 33 - Perú 448-0785

karinamoreno79@hotmail.com 